\title{
Is vegan feeding advisable in first childhood?
}

\section{Alicia Montiel del Canto ${ }^{1}$, Carmen Martín Salinas²*}

\footnotetext{
${ }^{*}$ Correspondence

Carmen Martin Salinas

Facultad de Medicina Arzobispo

Morcillo, 2-4 28029, Madrid - Spain

Email: carmen.salinas@uam.es

Telephone: 630451208

1School nurse. Madrid. Spain.

2Universidad Autónoma de Madrid,

Madrid, Spain.

Received: Feb 2, 2019

Accepted: Apr 19, 2019

Published: May 3, 2019

C2019 Canto A M del and Salinas C M; licensee OA Journal of Food and Nutrition. This is an Open Access article distributed under the terms of the Creative Commons Attribution License (http://creativecommons.org/licenses/ by/4.0), which permits unrestricted use, distribution, and reproduction in any medium, provided the original work is properly credited.
}

\begin{abstract}
Objective: To analyse if it is possible to correctly feed children with a vegan diet from birth.

Method: A two stages bibliographic search was conducted. The first one was in Google Academic Search, using the information obtained for the introduction. The second one in the Health Science Database PubMed, Cinhal and Scopus where 26 publications were chosen.
\end{abstract}

Results: The principal nutrient deficiencies that can be developed in vegan children were identified. Aferwards the growth curve of both vegan and omnivore children has been compared. In addition, we have clarified the nurses' knowledge about people's vegan nutritional requirements from birth. Finally, the benefits of following a vegan diet have been established.

Discussion and conclusions: well-planned vegan diets allow an adequate development of children from birth. The only compulsory nutrient supplementation is vitamin B12. The healthcare professionals have an important role since they have to give the guidelines to introduce the complementary feeding. Also, they have to inform the parents how to avoid the nutritional deficiencies that can give rise to serious health consequences.

Keywords: Infant, Child, Vegan diet, Vegetarianism, Nursing.

\section{Introduction}

Vegan parents try to implement vegan feeding in their children from birth. The World Health Organization (WHO) and the European Society for Paediatric Gastroenterology Hepatology and Nutrition (ESPGHAN) consider THAT this kind of feeding can develop a high risk of nutritional deficits that can lead to serious consequences in the kid 's health [1]. The vegan mothers that do not breastfeed, they tend to use soy or rice drinks as well as homemade formulas. However, the abovementioned drinks are not substitutives of the breastmilk [2].
Vegan and not vegan parents follow the same guides about introducing complementary feeding in their children [3]. It starts at sixth months of life. However, vegan parents should be informed from the healthcare professionals about this procedure in order to avoid on negative consequences on their children growth [4].

\section{Controversy about vegan/vegetarian diets}

Controversy, for there might be a lack of nutrients that are involved in important biological functions, especially during growth and development. All of them can be also included in a well-planned diet, excluding vitamin B12 [5]. 
Chart 1: Controversy of vegan diets in childhood.

\begin{tabular}{l|l}
$\begin{array}{l}\text { Benefits } \\
\text { Lower Body Mass Index } \\
\text { (BMI) }\end{array}$ & $\begin{array}{l}\text { Risks } \\
\text { nutrients: fatty acids, Fe, } \\
\text { Ca, Zn, I, Se, and } \\
\text { vitamins D, B12 and } \\
\text { riboflavin. }\end{array}$ \\
\hline $\begin{array}{l}\text { Reduce the incidence of } \\
\text { some type of cancers. }\end{array}$ & $\begin{array}{l}\text { Need more aliments to } \\
\text { cover the needs of } \\
\text { nutrients and energy. }\end{array}$ \\
$\begin{array}{ll}\text { Reduce the risk of } \\
\text { having heart diseases, } \\
\text { The benefits of vegan } \\
\text { diet since childhood, } \\
\text { obesity and type } 2 \\
\text { diabetes. }\end{array}$ & $\begin{array}{l}\text { Vegan kids tend to be } \\
\text { slightly skinny and } \\
\text { shorter than } \\
\text { omnivorous. }\end{array}$ \\
\hline
\end{tabular}

Western countries have conducted studies with vegan and vegetarian children and adolescents. They have showed That both of them have and development within the normal ranges According to standards population, although with a less Body Mass Index (BMI). Several official organisms have contradictory views about vegan diet. On the one hand, the Academy of Nutrition and Dietetics (AND) and the British Medical Association (BMA) [6] maintain that well-planned diets are appropriate for along all stages of life, including pregnancy, breastfeeding, infancy and childhood. On the other hand, the WHO and the ESPGHAN are more reticent about vegan diet in childhood because of the nutritional deficiencies that can cause if it is not well-planned. Even more, vegan diet has a high fibre content which can reduce the bioavailability of micronutrients and fatty acids and affect the normal childhood growth and development $[1,7]$.

Nevertheless, there are numerous studies which show The benefits of vegan diet since childhood [2].

The EPSGHAN published in 2017 a document which establish itself in complementary feeding. They suggest adopt vegan diets only under doctor 0 nurse's supervision in order to assure children correct levels of children receives enough vitamin B12, vitamin D, iron, zinc, folate, fatty acids, as well as, the nourishment provides the density of nutrients and energy needed are correctly provided with nourishment [1].
In this sense, an expert nutrition nurse's support may be beneficial for the parents. They should understand the bad consequences of not following the correct advices regarding the diet supplements. It is nurses right orientations throughout healthcare education that assures a right baby feeding [4].

The objective of this research is determining if vegan parents are conscious about the nutritional deficiencies that these diets can cause in their children but also the nutritional needs in every stage of life.

\section{Method}

A bibliographic research has been conducted under controlled language in Healthcare Databases PubMed, Cinahl and Scopus. English terms vegan diet, infant, child and vegetarianism were used in thesaurus (MeSH and DeCS), combined with boolean operators AND and OR.

The founded article selection was conducted from the title reading, followed by the abstract reading, and ending with a complete reading of the text.

\section{Test Results}

26 documents were chosen in the appendix 1 but only 4 categories which include different opinions about adopting a vegan diet from birth were selected.

\section{Compromised nutrients in childhood vegan diets}

Vegan diets can involve some risks in children's growth and development, concretely some nutritional déficits.

McEvoy and Woodside emphasised in a bibliographic research some nutrients which parents should pay more attention to. It includes an n-3 fatty acids, vitamin B12, which are exclusively obtained from animal origin handled, vitamin $\mathrm{D}$, calcium, due to the exclusion of daily products, and the presence of phytates and oxolates, which are vegetables. The research concludes that vegan diets in childhood require special attention to avoid nutritional deficiencies [10]. Ann Reed Mangels and Virginia Messina agree in their bibliographic research, that most of the problematic nutrients are vitamin B12, iron, zinc and vitamin D during the first six months. Since the second semester of life and previous to complementary feeding initiation, the progression 
patterns in feeding are equals as the non-vegans, paying special attention on allergenic nourishment, as can be dried fruit [11].

At the same time, Virginia Messina and Ann Reed Mangels [12] concluded in a posterior research that the nutrient intake in this type of diets is usually enough and sometimes exceed the omnivores one. They highlighted that, calcium levels are under the recommendations, similarly as well as vitamin B12 and, occasionally, vitamin D. Also, the zinc bioavailability can be affected by the phytates and blood level controls are necessary considered to avoid deficiencies. In addition, those researchers consider iron can adequate kids intake, even though the most part of the iron is non-hemo. However, there is not a high anemia incidence comparing with the rest of population. The intake of a mix of nonhemo iron and vitamin $C$ increase their bioavailability, making blood levels more adequate.

On the contrary, the German Nutrition Society (DGE) [13] opposed to vegan diet in childhood, adolescent, pregnancy and breastfeeding because of the vitamin B12. Deficiency, and its difficulty to find a not animal source extract of this vitamin. Considers, it is difficult to assure all necessary nutrients on this type of diet, especially in childhood, where nutritional deficiencies may cause serious kids health problems. According to the DGE, the ESPGHAN position is in favour of vegan diets, but maintaining strict conditions in assuring that an adequate suplementation of these considered "deficient nutrients" is supervised and made by healthcare profesionals. This would guarantee not only the correct kid's development, but also it would guide parents to understand the serious consequences of a diet without supplements [1].

The Academy of Nutrition and Dietetics (AND) appoints in favour of vegetarian diets, including vegans, but only if they are well-planned. AND considers them appropriate for every life stage and analyse the critical nutrients of this diets, including the following: proteins, n-3 fatty acids, iron, zinc, iodine, calcium, vitamin D and vitamin B12 [14]. AND highlights the support that healthcare professionals should provide families in terms of nutritional needs individually adapted [2].
A bibliographic research of Pawlak et all [15] about different kind of vegetarianism followed in different ethnic groups, assures that vegetarians develop vitamin B12 deficiency despite other factors, such as the demographic characteristic, residence country, age or type of vegetarian diet followed. On the contrary, Ambroszkiewicz found that vegetarian children had enough vitamin B12 in their diet (excluding vegans) and normal homocistein seric and folates concentrations, in unlike adults. He also underlined the need to monitor nutritients in this age to avoid future deficiencies [16]. In addition, Gorczyca et all conducted a study with 20 vegetarian kids and 20 omnivores, and concluded that iron intake in both groups was lower than what was recommended. However, iron deficiencies are frequent in vegetarian kids despite of having a high vitamin C intake [17]. Also, Sanchis- Chordá established was a research that the iron-deficiency anemia is similar in both vegetarians and omnivores [5]. On the contrary, Martinón et al [18] show a clinic case with a eight month baby from a ovo-vegetarian mother who is exclusively breastfeed. This baby have serious anemia, and low levels of vitamin D and B12, and a objective improve is shown after supplemented this nutrients.

Finally, Foster et all indicated that it's necessary to improve the methods level determination of this mineral to establish recommendations although the zinc deficiency is quite often. Likewise, he enounced that there is not enough data to concrete wheter zinc deficiencies are higher in omnivore than vegetarian kids in developed countries [19].

\section{Growing patterns evolution}

Most of the kids grow and develop normally, but they tend to be slightly skinny and smaller than the average. They have to carry out anthropometric controls of weight, height, and body mass index at least once per year. Also, a bone densitometry in adolescents has to be adopted to assure a good health status.

A Schürmann et all research, pointed out that weight, height and anthropometric measurement in babies, kids and adolescents, that follow a vegano $r$ vegetarian diet, are in the normal standards. It can occasionally be slightly lower in comparison with omnivores. He also comments, that the use of 
vegetarian diets from a therapeutic perspective, can prevent obesity in childhood due to the lower amount of fat and the lower weight of the kids that follow this diet [20]. In the same manner, Keller y Müller established that vegan children can grow under the normal standards, although they tend to be shorter and skinny [21].

Dunham and Kollar [22] established, that occasionally the growth ranges and calories intake are not adequate and indicated that these kids are under the normal standards; in those occasions it is necessary the intervention of healthcare professionals to avoid important nutritional deficiencies and a growth and development kids delay.

Huang et all conducted a study in 1151 vegetarian persons and concluded that these diets are beneficial to lose weight. However, their effects are likely to be reduced as time goes by [23].

ADA established that vegetarian children provided with good breast milk portion may have a normal growth [2]. In the same manner, The British Nutrition Foundation (BNF) established that kids vegan diets are low energy supplied, what makes these kids be frequently smaller. However, vegan mother's babies raised in veganism have a normal growth and development [6].

\section{Dietetic recommendations for vegetarian children. Healthcare professionals training needs.}

A well-planned vegan or vegetarian diet can reach nutritional objectives in different stage of life. However, not only is important to have the nutritional knowledge to choose the correct food combinations, but also to correctly plan these kinds of diets. In this sense, healthcare centre nurses have the privilege in early identification of growing deficiencies originated from a vegan feeding. The feeding habits surveys offer enough information to detect the problem. Furthermore, it guides the parents to mix food in vegan diet and value the need of supplements with shortfall nutrients.

McEvoy and Woodside [10] agreed with Virginia Messina and Ann Reed Mangels [12] research regarding the calcium, zinc and vitamin D supplements recommendations. For instance, to take vitamin B12 fortified aliments to avoid appearance of megaloblastic anemia from the complementary feeding beginning, provided that the vegan mother takes this vitamins during the lactation. SanchisChordá proposed to supplement vitamin B12 in kids since 1 to 3 years from the complementary feeding beginning with 5-20 $\mu \mathrm{g}$ up to 6 to 11 months $10-40 \mu \mathrm{g}$ [5].

In connection with calcium, the daily exclusion in vegan diets may represent a problem to cover the necessities of this mineral during childhood. Sanchis-Chordá [5] recommended, to drink two daily rations of non-daily drinks enriched with calcium to assure the correct calcium intake in vegan children. Furthermore, vitamin D deficiency is common among population because of sun exposure restriction due to the fear for suffering from skin cancer. However, it's important to know that vitamin D3, (included in egg yolk and fatty fish) is more efficient in reversing this vitamin deficiency. However, it exists supplements of vitamin D3 for vegans, which are normally removed from lichens.

The ESPGHAN'S position paper, established that it is strictly necessary that healthcare professionals track kids with these types of diets (mainly on babies that are still being breastfed) to assure the correct nutrient levels or the need of supplementation of some other nutrients in any mothers or babies [1].

Van Winckel et all [24] pointed out the importance of healthcare professionals in order to test and track the weight, growth and psychomotor development as part of the nutritional testing. They commented that vegan diets have to incorporate at least vitamin B12, but also to measure the adequate intake of iron, zinc, and aliments with energetic density and high-quality proteins. He also established that babies from 0 to 12 months must be supplemented with vitamin B12, regardless of whether the baby has been fed with soy formula or breast-feeding. He also recommends to start complementary feeding from 6 months but keeping breastfeed. The recommendations for 1-year old children are based to assure the calcium intake by supplemented drinks and the caloric density. Furthermore, not also is important to restrict the amount of raw foods because of their hard digestibility but also to propose parents to follow a weekly plan which allows a post evaluation. 
Mangels and Messina [11] favoured breastfeeding as the best option in vegan kids nutrition. Soy formulas are the only option in case breastfeeding is not possible, breastfeeding is usually recommended until 6 months. Afterwards it is progressively supplemented until 1 year of life. Vegan mothers who defend this type of diet used to continue breastfeeding after that time. However, parents can introduce fortified soy drinks after the first year of life. Vitamin B12 is the only strict supplementation, with $0,4 \mu \mathrm{g} /$ day during first 6 months and $0,5 \mu \mathrm{g} /$ day depart from this age. Zinc, vitamin D and iron are other supplements that may be needed.

The above-mentioned authors [11] recommended to start complementary feeding with fortified cereals, followed by fruits, juices and vegetables. At 7- or 8months age It starts being introduced protein source and ends with the insertion of pasta, rice, grains, bread among others. In this population group dried fruits are very important since are products frequently ingested by vegetarians. Also, they have a high allergen potential, so their incorporation should be postponed and need to be correctly monitored. Finally, they make a vegan kids menu purpose for 9 months babies, with three main meals and two snack options.

Messina and Mangels consider in their childhood vegan diets research that healthcare professionals should evaluate the diet to assure the intake of aliments with calcium, iron, zinc and vitamin B12. Vegan children should eat three meals per day but also they can eat two or three snacks in between. The growth slows down and hunger decrease from 18 months to 3 years; this stage is characterized by the food preference beginning and many kids show reticence to try new foods. The school kids incorporation requires special attention since shows the vegan children differences from the rest. That can make children reject vegan diet since it is different from their school mates.

Gibson et all [25] determined that the use of both iron and zinc fortified cereals increased their bioavailability in the organism despite the presence of absorption inhibitors such as phytates and polyphenols. Zinc supplemented recommendations are $5 \mathrm{mg} /$ day in 6 to 36 months kids and $10 \mathrm{mg} /$ day in kids older than 3 years. In iron's case, the recommendation is $2 \mathrm{mg} / \mathrm{kg}$ per day for 6 to 23 months children and 20-60 mg/day for kids older than 2 years.

Hood [26] enhanced the nursing important role when focused on are parent's education focused on are follow a vegan diet. The main issues that nurses should be focused on are to assure the vitamin B12 diet content (both by fortified food or supplementation) but also iodine adequate intake and the eating of calcium and n-3 fatty acids aliments and finally changing the aliments in order to make food more interesting by colours and different textures. Along the same line Mangels and Driggers [27] highlighted the healthcare professional's important role when advising during the children growth but also helping families to choose the food correctly. Also, they evaluate the supplementation need and help them with meal schedule, proposing a vegan or vegetarian children's menu for 2 years old. In respect of vitamin D, they establish that a 30 minutes weekly solar exposure is enough to have the necessary vitamin D amount required.

Moilanen [28] recommended vitamin B12 supplementation in all the babies, regardless of whether they are breastfeeding and their mothers supplement this vitamin, or they are feed with soy formula. Related with zinc, he advises to improve their bioavailability with soy fermented products as tempeh or brown bread with fermented leaven. Also, calcium levels can be affected and he recommends soy drinks or enriched orange juices, with high calcium level aliments such as broccoli, kale or curly kale.

It is necessary to take a strict control by nurses in case adolescents decide to adopt a vegan diet. In some cases, the main object is to lose weight but it can lead to eating disorders [28]. Hood shows that more than a half of the people with such eating disorders has follow a vegan or vegetarian diet to lose fat and erase caloric products and dairy in their diets. However, the recovery from these disorders is similar, regardless of vegan, vegetarian or omnivore diet [26].

Pawlack et all 's [15] vitamin B12 deficiencies research recommended a strict control of this vitamin during the human being development. The main reason is because some people continued having those 
deficiencies despite of the diet supplementation. This is mainly caused by two factors: on the one hand they eat them with less frequency than it is recommended. On the other hand, they ingest a lower dose than the one their bodies need to sustain adequate levels.

Recently, Baroni et al have designed the VegPlate Junior (VPJ), a specific guide for the vegan dietetic planning in childhood and adolescent [29], with the aim of guide the healthcare professionals to advise vegetarian parents about well dietetic patterns for their children. This guide obeys the criteria of wellplanned nourishing and adequate for the normal growth promotion. Same author [30] sums up the Vegetarian Scientific Society recommendations concluding that well planned vegan diets and based on a huge variety of vegetal aliments can be an adequate nutrition for all life stages, including pregnancy, breastfeed, childhood and adolescence.

\section{Vegan diet benefits}

The benefits of veganism start from the understanding that people who choose this diet pattern conduct a healthier life than the omnivores. Vegan people used to have better health and less risk of having cardiovascular diseases and some types of cancer. It has been recently observed that there is a close relation between vegan diet and microbiota. Some microorganisms and metabolites that we have in our intestine play an important role in our health [5].

The American Dietetic Association (ADA), together with the Canadian dieticians, are in favour of vegan and vegetarian diets. They consider them adequate for all life stages but also beneficial to health and it helps preventing from diseases as obesity, heart diseases, hypertension, diabetes, cancer, osteoporosis, renal disease or dementia. They highlight the existence of control programs for this type of diet but also to take actions by healthcare professionals in order to carry on nutrional monitoring [2, 31]. Similarly, the British Nutrition Foundation (BNF) pointed out the health benefits potential of vegetarian and vegan diets from childhood since it is related with lower mortality, heart disease, cancer and osteoporosis [6]. The Academy of Nutrition and Dietetics (AND) also showed the potential benefits of these diets with obesity and overweight, heart diseases, hyperlipidemic, hypertension, diabetes, cancer and osteoporosis [13].

Lee et al [32] conducted a 2 diabetes glycemic control research comparing vegan and conventional diets. He concluded that vegan people have a better glycemic control against those who follow the Korean diabetes association dietetic recommendations.

Mediterranean diet adds countless benefits to improve cardiovascular health and diseases. Sofi et all [33] proposed a study protocol comparing vegetarian and Mediterranean diets in order to know which diet had the best results against cardiovascular diseases.

Huang et all studied the relation between different vegetarian diets and weight control. That study highlighted that these diets are good to prevent obesity. However, vegan diet appears to result in greater weightloss [23]

Tantamango-Bartley et all [34] conducted a study among the vegetarianism influence in having a less risk of cancer in the population. The study concluded that vegetarian diets offer a better protection against cancer, being vegan diets the ones with not only having less risk of sufferinf from cancer in general but also in preventing some specific women cancers.

\section{Discussion}

The review conducted on vegan diets in childhood indicates that, despite the high nutritional needs in this stage of life, if they are well planned, they include a wide variety of plant foods and supplements of vitamin B12 and vitamin D. Nutrient deficits would disappear and these diets can be tracked from birth, childhood until adolescence, allowing proper growth and development, although there is no unanimous consensus on the nutrients that need to be supplemented, with the exception of vitamin B12 [10, 11, 13-19, 24, 25, 28, 30], being necessary to maintain strict vigilance to avoid possible nutrient deficits.

In relation to growth and body weight [20-23], most children grow and develop normally, but tend to be thinner and smaller than the average of the population because vegan diets are generally low in energy, which makes these children often smaller. 
The nurse should evaluate in the follow-up babies consultation, all the indicators related to growth and development from birth and for short periods of time. At six months, coinciding with complementary feeding, the identification of vegan parents who want to establish this type of feeding in their children is of special interest. At this point, it is important to listen to the very description of the diet that families make, vegan and omnivorous, to determine which foods can play a key role in their menus planning In order to understand the information obtained from families, it is necessary that nurses have a reliable source of knowledge that allows them to inform and guide these families, to avoid nutritional deficiencies or food errors that may affect growth and the development of their children as a result of an incorrect feeding. It is important to emphasize that the families themselves rely on health professionals as advisors of a healthy diet for their children development [1, 5, 12, 26-29].

Also, vegan diets, because of their high fiber content and antioxidant nutrients, have been shown to prevent obesity in childhood and adolescence, as well as diseases related to it, such as dyslipidemia, high blood pressure, cardiovascular diseases and some types of cancer, in later stages of life $[2,5,6,13$, 22, 31-34].

\section{Conclusions}

Properly planned vegan diets are a valid option in all stages of life, including pregnancy, lactation, childhood and adolescence.

Families that choose this type of food should be advised by expert nurses to avoid nutritional deficiencies at critical times of growth.

When children get used to take a wide variety of plant foods from the very first years of life, it promotes healthy eating habits that will last a lifetime.

\section{Recognition}

We appreciate the unselfish collaboration of Professor Isabel Guzmán in the translation of this article.

\section{References}

1. Fewtrell M, Bronsky J, Campoy C, et al. (2017) Complementary Feeding: A Position Paper by the European Society for Paediatric Gastroenterology, Hepatology, and Nutrition (ESPGHAN) Committee on Nutrition. JPGN. 64:119-132.
2. Craig WJ, Mangels AR (2009) American Dietetic Association Position of the American Dietetic Association: vegetarian diets. J Am Diet Assoc. 109:1266-1282.

3. Saz-Peiró P, Morán Del Ruste M, Saz-Tejero S. (2013) La dieta vegetariana y su aplicación terapéutica. Medicina Naturista. 7:13-27.

4. Adrada Trujillo E, Álvarez Gómez J, Arana Cañedo-Argüelles C, et al. (2017) Guías Conjuntas de Patología Digestiva Pediátrica Primaria-Especializada del Grupo de Gastroenterología Pediátrica de la Zona Sur-Oeste de Madrid. Aepap. 2019:2-12.

5. Sanchis-Chordà J, Redondo-Cuevas L, Codoñer-Franch P (2016) Dieta vegana en la infancia: beneficios y riesgos. Rev Esp Pediatr. 72:299-303

6. Phillips F (2008) Vegetarian nutrition. Br Nutr Found Nutr Bull. 30:132-167

7. Daza W, Dadán S. (2019) Alimentación complementaria en el primer año de vida. Programa de Educación Continua en Pediatría - PRECOP - Sociedad Colombiana de Pediatría [Internet]. 8:18-27.

8. Key TJ, Appleby PN, Davey GK, et al. (2003) Mortality in British vegetarians: review and preliminary results from EPIC-Oxford. Am J Clin Nutr. 78:533S-538S

9. López Portillo L, García Campos M, Montijo Barrios E, et al. (2006) La dieta vegetariana en los niños. Ventajas, desventajas y recomendaciones dietéticas. Acta Pediatr Mex [Internet] 27:205-212

10. McEvoy CT, Woodside JV. (2015) 2.9 Vegetarian diets. World Rev Nutr Diet. 113:134-138

11. Mangels AR, Messina V (2001) Considerations in planning vegan diets: infants. J Am Diet Assoc. 101:670-677.

12. Messina V, Mangels AR (2001) Considerations in planning vegan diets: children. J Am Diet Assoc. 101:661-669.

13. Richter M, Boeing H, Grünewald-Funk D, et al. (2016) Vegan Diet. Position of the German Nutrition Society (DGE). Ernaehrungs Umschau Int. 63:92-102.

14. Melina V, Craig W, Levin S (2016) Position of the Academy of Nutrition and Dietetics: Vegetarian Diets. I Acad Nutr Diet. 116:1970-1980

15. Pawlak R, Parrott SJ, Raj S, et al. (2013) How prevalent is vitamin B12 deficiency among vegetarians? Nutr Rev. 71:110-117.

16. Ambroszkiewicz J, Klemarczyk W, Chełchowska M, et al. (2006) Serum homocysteine, folate, vitamin B12 and total antioxidant status in vegetarian children. Adv Med Sci. 51:265-268.

17. Gorczyca D, Prescha A, Szeremeta K, et al. (2013) Iron status and dietary iron intake of vegetarian children from Poland. Ann Nutr Metab. 62:291-297.

18. Martinón TN, Crujeiras MV, Saborido FR, et al. (2018) Anemis grave y malnutrición en hijo de madre vegetariana y lactancia materna prolongada. Rev Esp Nutr Comunitaria. 24:168.

19. Foster M, Samman S (2015) Vegetarian diets across the lifecycle: impact on zinc intake and status. Adv Food Nutr Res. 74:93-131.

20. Schürmann S, Kersting M, Alexy U (2017) Vegetarian diets in children: a systematic review. Eur J Nutr. 56:1797-1817.

21. Keller M, Müller S (2016) Vegetarische und vegane Ernährung bei Kindern - Stand der Forschung und Forschungsbedarf Forsch Komplementmed. 23:81-88.

22. Dunham L, Kollar LM (2006) Vegetarian eating for children and adolescents. J Pediatr Health Care. 20:27-34.

23. Huang RY, Huang CC, Hu FB, et al. (2016) Vegetarian Diets and Weight Reduction: a Meta-Analysis of Randomized Controlled Trials. J Gen Intern Med. 31:109-116.

24. Van WM, Vande VS, De BR, et al. (2011) Clinical practice: vegetarian infant and child nutrition. Eur J Pediatr 170:1489-1494. 
25. Gibson RS, Heath AL, Szymlek-Gay EA (2014) Is iron and zinc nutrition a concern for vegetarian infants and young children in industrialized countries? Am J Clin Nutr. 100:459S-468S.

26. Hood S (2008) The vegan diet. PRACT NURSE 35:13-17.

27. Mangels R, Driggers J (2011) The Youngest Vegetarians: Vegetarian Infants and Toddlers. ICAN: Infant, Child, \& Adolescent Nutrition. 4:8-20.

28. Moilanen BC (2004) Vegan diets in infants, children, and adolescents. Pediatr Rev. 25:174-176.

29. Baroni L, Goggi S, Battino M (2018) Planificación de las dietas vegetarianas bien equilibradas en lactantes, niños y adolescentes: el Vegplate junior. J. Academia de Nutrición y Dietética. 119:3.

30. Baroni L, Goggi S, Battino M (2019) Vegan Nutrición para madres y niños: herramientas prácticas para proveedores de atención médica. Nutrientes. 11:5.
31. American Dietetic Association; Dietitians of Canada. (2003) Position of the American Dietetic Association and Dietitians of Canada: Vegetarian diets. J Am Diet Assoc. 103:748-765.

32. Lee YM, Kim SA, Lee IK, et al. (2016) Effect of a Brown Rice Based Vegan Diet and Conventional Diabetic Diet on Glycemic Control of Patients with Type 2 Diabetes: A 12-Week Randomized Clinical Trial. PLoS One. 2:e0155918.

33. Sofi F, Dinu M, Pagliai G, et al. (2016) Mediterranean versus vegetarian diet for cardiovascular disease prevention (the CARDIVEG study): study protocol for a randomized controlled trial. Trials. 17:233

34. Tantamango-Bartley Y, Jaceldo-Siegl K, Fan J, et al. (2013) Vegetarian diets and the incidence of cancer in a low-risk population. Cancer Epidemiol Biomarkers Prev. 22:286-294. 